\title{
Duals for Classical Inventory Models via Generalized Geometric Programming
}

\author{
CARLTON H. SCOTT $\dagger$ \\ University of California Irvine, Graduate School of Management, Irvine, California \\ 92697 USA \\ THOMAS R. JEFFERSON \\ University of Florida, Gainesville, Warrington College of Business, Decision and In- \\ formation Sciences Department, Gainesville, Florida 32611 USA \\ SOHEILA JORJANI \\ California State University San Marcos, College of Business, High Technology Man- \\ agement Department, San Marcos, CA 92096 USA
}

\begin{abstract}
Inventory problems generally have a structure that can be exploited for computational purposes. Here, we look at the duals of two seemingly unrelated inventory models that suggest an interesting duality between discrete time optimal control and optimization over an ordered sequence of variables. Concepts from conjugate duality and generalized geometric programming are used to establish the duality.
\end{abstract}

Keywords: Inventory models, conjugate duality, generalized geometric programming.

\section{Introduction}

Geometric Programming is now a well-established branch of optimization theory and has been instrumental in the solution of many nonlinear optimization problems occurring in such diverse areas as marketing, Balachandran and Gensch [1], water pollution management, Ecker and McNamara [4] production engineering, Petropoulos [8], transportation planning, Jefferson and Scott [9], and machine maintenance, Cheng [2]. Initially, geometric programming concerned itself with finite dimensional optimization problems where both the objectives and the constraints were in posynomial form (i.e. polynomial with positive coefficients). Subsequently the theory was extended by Peterson [7] to any finite dimensional convex programming problem. This latter development is termed generalized geometric programming and it is with the so called "unconstrained" version of generalized geometric programming that we will be concerned with in this

$\uparrow$ Requests for reprints should be sent to Carlton Scott, University of California Irvine, Graduate School of Management, Irvine, California 92697 USA. 
paper. Various other extensions are possible such as to composite geometric programming, Jefferson and Scott and Wang [10] which have particular application in tool replacement strategies, Scott, Jefferson and Lee [13].

Generalized geometric programming is novel in its approach to mathematical programming in that it identifies and utilizes the following properties: (i) convexity, (ii) linearity, (iii) separability, and (iv) duality. Convexity and linearity are two very important functional forms in mathematical programming and they provide powerful results regarding optima. In addition, powerful computational techniques are associated with these forms. Separability, even if only partial, provides insight into the solution of a mathematical program and often contains the key to a successful decomposition. The dual problem, taken in the geometric programming sense often provides a much simpler problem to work with for the following two reasons:

(i) the dual problem is optimized over the polar to the cone constraints in the primal. This means that if the primal cone is of high dimension, the polar cone is of low dimension - a computational bonus!

(ii) the constraints in the primal problem are absorbed into the dual objective function. In this way, while the primal program may have nonlinear constraints, the dual may be simply an optimization over a polyhedral set.

Joint utilization of the primal and dual objectives yields a powerful algorithmic stopping criteria; i.e. the sum of both objectives is less than some specified tolerance. Finally, the dual often gives a different perspective to the problem which has useful interpretational insights. Many examples are given in Peterson [7] and focused applications on location theory may be found in Scott, Jefferson and Jorjani [12].

As stated earlier, we will be concerned with the so called "unconstrained" version of generalized geometric programming which in conventional terms means a finite dimensional convex programming problem with the constraint set being a cone. Reason (ii) above refers to the constrained theory [7]. In this paper, we present the theory pertaining to this particular problem type and then we look at the application to two problems of inventory control, Clark and Scarf [3], Modigliani and Hohn [5]. These seemingly unrelated models are in fact, related in the sense of duality. 


\section{Generalized Geometric Programming}

We consider an optimization problem of the form:

$$
\text { Minimize } f(x) \text { over } x \in \mathrm{C} \cap \chi
$$

where $\chi$ is a cone in $\mathrm{E}_{n}$ and $f$ is a closed convex function with domain $\mathrm{C}$. This problem is termed the primal problem. We associate with the primal problem, another problem, called the dual problem, which is of the form:

$$
\text { Minimize } g(y) \quad \text { over } y \in \mathrm{D} \cap \chi^{*}
$$

where $\chi^{*}$ denotes the polar cone of $\chi$ in $\mathrm{E}_{n}$ and $[g: \mathrm{D}]$ is the conjugate transform of $[f: \mathrm{C}]$ with value

$$
\begin{gathered}
g(y)=\sup _{x \in C}\left(x^{T} y-f(x)\right), \\
D=\left\{y \in E_{n} \mid x^{T} y-f(x)<\infty\right\}
\end{gathered}
$$

and

$$
\chi^{*}=\left\{y \mid x^{T} y \geq 0 \quad \forall x \in \chi\right\}
$$

We note that the primal and dual problems are essentially of the same form and hence are termed symmetric. At optimality, the following relationships hold between the primal the primal and dual optimal points, $x_{0}$ and $y_{0}$ respectively.

$$
\begin{aligned}
& f\left(x_{0}\right)+g\left(y_{0}\right)=0 \\
& y_{0} \in \partial f\left(x_{0}\right), x_{0} \in \partial g\left(\mathrm{y}_{0}\right) \\
& x_{0}^{T} \mathrm{y}_{0}=0
\end{aligned}
$$

These optimality conditions allow an optimal point for one program to be calculated from an optimal point of the other. Here $\partial f\left(x_{0}\right)$ denotes the subgradient set of $f$ at $x_{0}$. That is,

$$
\partial f\left(x_{0}\right)=\left\{y \mid f\left(x_{0}\right)+<y, x-x_{0}>\leq f(x), \forall x \in C\right\}
$$

For full details of the theory given in this section the reader is referred to Peterson [7]. 


\section{A Multi-Echelon Inventory Model}

For a storage network, with $n$ facilities in series, uncertain demand D, occurs at facility $n$ only. Shortages are passed up the line to $n-1$, then $n-2$ if $n-1$ is out of stock, etc. Facility $n$ may be a retail store, whereas facility 1 may be a distant warehouse where storage costs are much lower.

Let $u_{i}$ be the sum of stock on hand at facilities $i, i=1, \ldots, n$. Hence

$$
u_{1} \geq u_{2} \geq \ldots \geq u_{n}
$$

At the beginning of a planning period, each facility places an order with immediate delivery. Let $c_{i}$ denote the amount by which the unit capital charge on ordering at facility $i$ exceeds that at $i-1$ and $c_{0}=0$. Similarly let $h_{i}$ denote that amount by which the unit storage cost at facility $i$ exceeds that at facility $i-1$ and $h_{0}=0$. We note that $c_{i} \geq 0, h_{i} \geq 0$ is reasonable since distant warehouses may have smaller holding and ordering costs than retail stores within a city. Let $p_{i}$ denote the unit shortage cost at facility $i$. Hence the total costs incurred are

$$
\sum_{i} c_{i} u_{i}+\sum_{i=1}^{n}\left(p_{i} E\left[D-u_{i}\right]^{+}+h_{i} E\left[u_{i}-D\right]^{+}\right)
$$

which represent the ordering cost, expected shortage cost and expected holding cost respectively and $\zeta^{+}=\max [\zeta, 0]$. Hence we obtain the following optimization problem: minimize the convex function

$$
\sum_{i}\left(c_{i}-p_{i}\right) u_{i}+\sum_{i}\left(p_{i}+h_{i}\right) E\left[u_{i}-D\right]^{+}+\sum_{i} p_{i} E[D]
$$

subject to equation (10),

$$
u_{i} \geq u_{i+1} \mathrm{i}=1,2, \ldots, \mathrm{n}-1
$$

In the generalized geometric programming form, we have to minimize

$$
\sum_{i} a_{i} u_{i}+b_{i} \int_{D=0}^{D=u_{i}}\left(u_{i}-D\right) f(D) d D
$$

subject to the cone condition

$$
P u \geq 0
$$


where

$a_{i}=\left(c_{i}-p_{i}\right), b_{i}=\left(p_{i}+h_{i}\right), u=\left(u_{1}, \ldots, u_{n}\right)^{T}$ and $P$ is an $(n-1) \times n$ matrix with elements $p_{i j}=+1$ for $i=j$ and $p_{i j}=-1$ for $j=i+1$. $f$ denotes the probability density function of demand. For the dual problem, we require the conjugate transform of the objective function, equation (14), and polar of equation (15). Taking the conjugate transform defined by equations (3), of (14) results in the supremum being attained when

$$
v_{i}-a_{i}-b_{i} F\left(u_{i}\right)=0
$$

where $v_{i}$ is the dual variable corresponding to $u_{i}$ and $F$ is the cumulative distribution function of demand. Hence, solving equation (16) for $u_{i}$ we have that

$$
u_{i}=F^{-1}\left(\left(v_{i}-a_{i}\right) / b_{i}\right)
$$

Hence the dual objective function is

$$
\begin{aligned}
\sum_{i}\left(v_{i}\right. & \left.-a_{i}\right) F^{-1}\left(\left(v_{i}-a_{i}\right) / b_{i}\right)-b_{i} \int_{D=0}^{\hat{D}}\left(F^{-1}\left(\left(v_{i}-a_{i}\right) / b_{i}\right)-D\right) f(D) d D \\
& -\sum_{i} p_{i} E[D]
\end{aligned}
$$

where $\hat{D}=F^{-1}\left(\left(v_{i}-a_{i}\right) / b_{i}\right)$.

The polar cone is given by

$$
\left\{v \mid v^{T} u \geq 0 \quad \forall u \text { s.t. } P u \geq 0\right\}
$$

Equation (18) readily implies that

$$
v=P^{T} \mathrm{z}, \mathrm{z} \geq 0
$$

where $z$ is an $n-1$ dimensional vector.

Expanding equation (19), we have that

$$
\begin{aligned}
& v_{1}=\mathrm{z}_{1} \\
& v_{i}=\mathrm{z}_{i}-\mathrm{z}_{i-1} \quad i=2, \ldots, n-1 \\
& v_{n}=-\mathrm{z}_{n-1}
\end{aligned}
$$

We note that the dual problem, i.e. the minimum of equation (16) subject to equations (20) and $\mathrm{z} \geq 0$ is a discrete time optimal control problem, 
where $\mathrm{z}_{i}, i=1, \ldots, n-1$ are the state variables and $v_{i}, i=1, \ldots, n$ are the control variables.

Several other models which fit into this framework may be found in Muckstadt and Roundy [6] and a specialized algorithm has been given by Scott and Tang [11].

A numerical example follows:

Suppose the demand D follows an exponential distribution with mean $\lambda$. Hence

$$
\begin{aligned}
& f(D)=1 / \lambda \exp (-D / \lambda), F(u)=1-e^{-D / \lambda} \\
& \text { and } F^{-1}(\alpha)=-\lambda \ln (1-\alpha)
\end{aligned}
$$

It follows that the primal objective given by equation (16) is

$$
\sum_{i}\left(\left(a_{i}+b_{i}\right) u_{i}+b_{i} \lambda e^{-u_{i} / \lambda}-h_{i} \lambda\right)
$$

The corresponding dual objective is from equation (19)

$$
\sum_{i}\left(\lambda\left(a_{i}+b_{i}-\nu_{i}\right) \ln \left(\frac{a_{i}+b_{i}+\nu_{i}}{b_{i}}\right)-c_{i} \lambda+\nu_{i} \lambda\right)
$$

where the primal and dual variables are related by

$$
u_{i}=-\lambda \ln \left(\frac{a_{i}+b_{i}-\nu_{i}}{b_{i}}\right) \quad \mathrm{i}=1, . ., \mathrm{n}
$$

We now particularize to the following data set.

$n=3, \lambda=100, c=(25,60,40), h=(10,20,30),, p=(50,100,80)$. In this case, the minimal cost is 20798.15 with optimal dual variables $\nu=$ $(0,1.739,-1.739)$ and corresponding primal variables $u=(53.9,42.7,42.7)$.This implies that it is optimal to hold 42.7 units of inventory at facility 3 and the remaining 11.2 at facility 1 .

\section{An Inventory-Production Control Model}

A well-known problem in inventory control is to select a set $\left\{x_{t} \geq 0 t=\right.$ $1, \ldots, T\}$ of production levels to minimize, over a planning horizon of length 
$\mathrm{T}$, the sum of production and holding costs, while meeting demand. Formally the problem may be posed as:

$$
\operatorname{Minimize} \sum_{\mathrm{t}=1}^{\mathrm{T}}\left(c\left(x_{t}\right)+h_{t} y_{t}\right)
$$

subject to the inventory balance dynamics

$$
\begin{aligned}
& y_{1}=x_{1}-d_{1} \\
& y_{t}-y_{t-1}=x_{t}-d_{t} t=2, \ldots, T-1 \\
& -y_{T-1}=x_{T}-d_{T}
\end{aligned}
$$

and the non-negativity constraints

$$
\begin{aligned}
& y_{t} \geq 0 t=1, \ldots, T \\
& x_{t} \geq 0 t=1, \ldots, T
\end{aligned}
$$

Here $y_{t}$ denotes the inventory level in period $t, d_{t}$ is the demand in period $\mathrm{t}, c\left(x_{t}\right)$ is the production cost (assumed convex and strictly monotonically increasing) and $h_{t}$ is the holding cost per unit in period t.

To invoke the theory of generalized geometric programming, we need to put the constraint equations (22) and (23) into a cone. Hence we introduce a new variable $\alpha_{t}, t=1, \ldots, T$ and restrict it to a one point domain $\left\{d_{t}\right\}, t=1, \ldots, T$. This variable is then associated with an additive component of the objective function which is identically zero. Hence we obtain a cone condition

$$
\begin{aligned}
& y_{1}-x_{1}+\alpha_{1}=0 \\
& y_{t}-y_{t-1}-x_{t}+\alpha_{t}=0 t=2, \ldots, T-1 \\
& -y_{T-1}-x_{T}+\alpha_{T}=0 \\
& y_{t} \geq 0 t=1, \ldots, T
\end{aligned}
$$

It is convenient to treat the other non-negativity constraint $x_{t} \geq 0, \mathrm{t}=$ $1, \ldots, T$ as an implicit one.

Our problem is now in a form which is directly suitable for application of the theory. The dual objective is given by

$$
\begin{aligned}
& \sup _{x_{t}, y_{t}, \alpha_{t}} \sum_{t}\left(x_{t} u_{t}+y_{t} v_{t}+\alpha_{t} \beta_{t}-c\left(x_{t}\right)-h_{t} y_{t}\right) \\
& x_{t} \geq 0 \\
= & \sup _{x_{t} \geq 0} \sum_{t}\left(x_{t} u_{t}-c\left(x_{t}\right)\right)+\sup _{\alpha_{t}} \sum_{t} \alpha_{t} \beta_{t}+\sup _{y_{t}} \sum_{t}\left(y_{t} v_{t}-h_{t} y_{t}\right) \\
= & \sum_{t} c^{*}\left(u_{t}\right)+d_{t} \beta_{t}
\end{aligned}
$$




$$
\begin{aligned}
& \text { where } c^{*}\left(u_{t}\right)=\sup _{x_{t} \geq 0}\left(x_{t} u_{t}-c\left(x_{t}\right)\right) \\
& u_{t} \in \partial c\left(x_{t}\right) \text { and } v_{t}=h_{t}
\end{aligned}
$$

Usually $c\left(x_{t}\right)$ is a quadratic function and $c^{*}\left(u_{t}\right)$ is readily calculated. Further we require the polar to the cone defined by equations (24) i.e.

$\left\{\left(u_{t}, v_{t}, \beta_{t}\right) \mid \sum_{t}\left(u_{t} x_{t}+v_{t} y_{t}+\beta_{t} \alpha_{t}\right) \geq 0 \forall x_{t}, y_{t}, \alpha_{t}\right.$ satisfying equations (26) $\}$

A straightforward calculation shows that

$$
\begin{aligned}
& v_{t}=p_{t}-p_{t+1}+q_{t} t=1, \ldots, T-1 \\
& u_{t}=-p_{t} t=1, \ldots, T \\
& \beta_{t}=p_{t} t=1, \ldots, T \\
& q_{t} \geq 0 t=1, \ldots, T
\end{aligned}
$$

Hence the dual problem is to minimize

$$
\sum_{t=1}^{T}\left(c^{*}\left(-p_{t}\right)+d_{t} p_{t}\right)
$$

subject to

$$
p_{t}-p_{t+1}+h_{t} \leq 0 \mathrm{t}=1, \ldots, \mathrm{T}-1
$$

For constant $h_{t}$, we have a minimization over a monotonically increasing set of decision variables.

A numerical example follows:

Suppose $c\left(x_{t}\right)=4 x_{t}^{2}$ for $T=3$ and $h=(10,5), d=(100,200,300)$. In this case, $c^{*}\left(p_{t}\right)=p_{t}^{2} / 16$ with $p_{t}=8 x_{t}$.

The primal program is

$$
\begin{aligned}
& \text { Minimize } \sum_{t=1}^{3} 4 x_{t}^{2}+10 y_{1}+5 y_{2} \\
& \text { subject to: } \\
& y_{1}=x_{1}-100 \\
& y_{2}-y_{1}=x_{2}-200 \\
& -y_{2}=x_{3}-300 \\
& \text { and } x \geq 0, y \geq 0
\end{aligned}
$$


The corresponding dual program is:

$$
\begin{gathered}
\text { Minimize } \sum_{t=1}^{3} \frac{1}{16} p_{t}^{2}+100 p_{1}+200 p_{2}+300 p_{3} \\
\text { subject to : } \\
p_{1}-p_{2}-10 \leq 0 \\
p_{2}-p_{3}-5 \leq 0
\end{gathered}
$$

Solving the dual program yields the optimal value of $-481,492.7$ with optimal solutions $p=(-1591.67,-1601.67,-1606.67)$. The corresponding primal program has minimal cost 481,492.7 with optimal solution (rounded) $x=(199,200,201)$ and $\mathrm{y}=(99,99)$.

\section{Conclusions}

The two inventory problems considered suggest an interesting duality between certain problems of discrete time optimal control and static optimization over monotonic sequences. The multi-echelon inventory model considered in Section 3 is a static optimization over monotonic sequences and the geometric dual is a discrete time optimal control problem. The reverse is the case for the production planning problem treated in Section 3. Problems of both type arise naturally in inventory control. Further, with a dual problem we are in a far better position to develop efficient computational algorithms that if only the primal is considered.

\section{References}

1. Balachandran, V. and Gensch, H. (1974) Solving the Marketing Mix Problem Using Geometric Programming, Management Science, 21, 160-171.

2. Cheng, T.C. (1992) Optimal Replacement of Aging Equipment using Geometric Programming, International Journal of Production Research, 30, 2151-2158.

3. Clark, A. and Scarf, H. (1960) Optimal Policies for a Multi-Echelon Inventory Problem, Management Science, 6, 475-490.

4. Ecker, J.G. and McNamara, J.R. (1971) Geometric Programming and the Preliminary Design of Industrial waste Treatment Plants, Water Resources Research, 7, $18-22$.

5. Modigliani, F. and Hohn, F. (1955) Production Planning Over Time Nature of The Expectation and Planning Horizon, Econometrica 23, 46-66.

6. Muckstadt, J. and Roundy, R. (1993) Analysis of Multistage Production Systems. In Handbooks in OR \&MS. S.C. Graves et al., eds. North Holland, Amsterdam, 59-131.

7. Peterson, E.L. (1976) Geometric Programming, S.I.A.M. Review, 18, 1-52.

8. Petropoulos, P.G. (1973) Optimal Selection of Machining Rate Variables by Geometric Programming, International Journal of Production Research, 11, 305-314. 
9. Scott, C.H. and Jefferson, T.R. (1978) Entropy and Geometric Programming in Transportation Planning, Opsearch, 15, 22-34.

10. Scott, C.H., Jefferson, T.R., and Wang, Y.P. (1990) Composite Geometric Programming, Journal of Optimization Theory and Applications, 64, 101-118.

11. Scott, C.H. and Tang, C.S. (1992) Separable Convex Programs with Ratio Constraints over a Directed Tree, Operations Research Letters, 11, 147-152.

12. Scott, C.H., Jefferson, T.R., and Jorjani, S. (1995) Conjugate Duality in Facility Location. In Facility Location: A Survey of Applications and Methods, Z. Drezner ed. Springer Verlag, New York, 89-101.

13. Scott, C.H., Jefferson. T.R., and Lee, A. (1996) Stochastic Tool Management via Composite Geometric Programming, Optimization, 36, 59-74. 


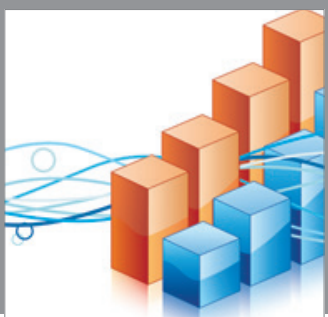

Advances in

Operations Research

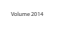

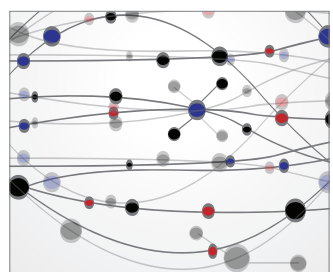

\section{The Scientific} World Journal
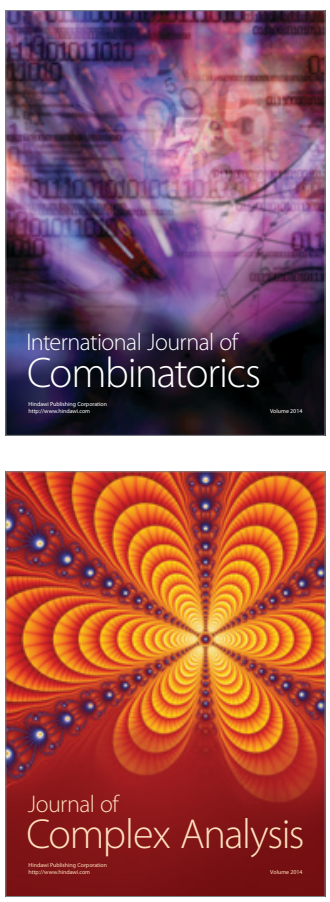

International Journal of

Mathematics and

Mathematical

Sciences
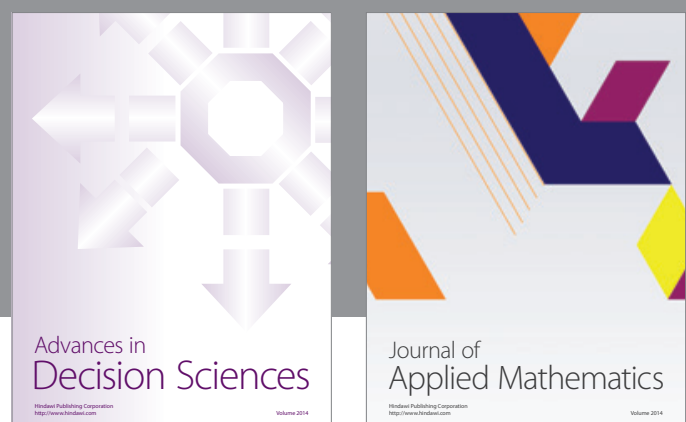

Journal of

Applied Mathematics
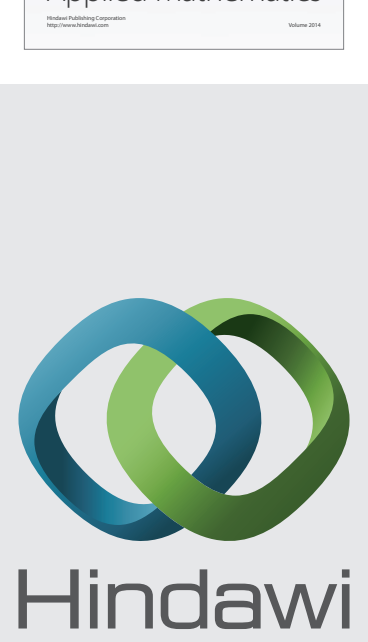

Submit your manuscripts at http://www.hindawi.com
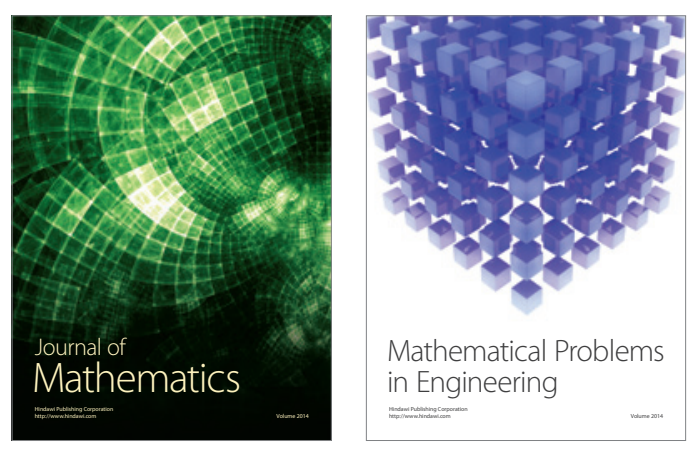

Mathematical Problems in Engineering
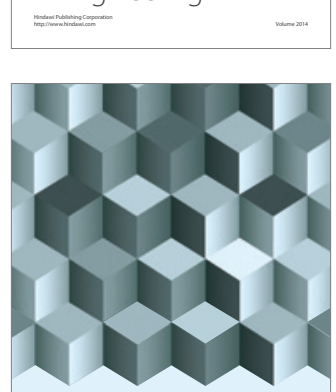

Journal of

Function Spaces
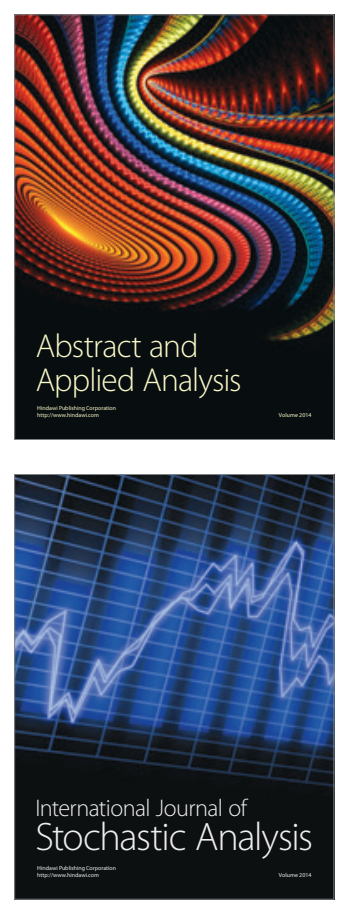

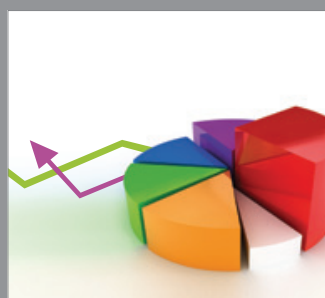

ournal of

Probability and Statistics

Promensencen
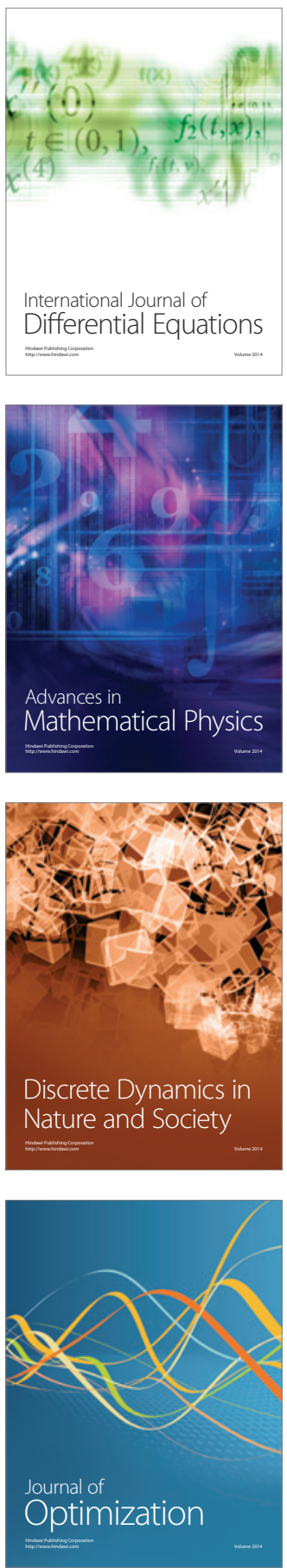